\title{
Development of Abdominal Compartment Syndrome in an 82-Year-Old Female Patient Immediately Following a Right Hip Arthroplasty
}

\author{
Remone Yousif', Fenghua Li', Reza Gorji', Syed Ali', Timothy Damron² \\ and Zhong-J in Yang ${ }^{1 *}$ \\ Department of Pharmaceutical Sciences, College of Pharmacy, Nova Southeastern University, USA
}

\begin{abstract}
Received: November 22, 2013; Accepted: December 10, 2013, Published: December 19, 2013
*Corresponding author: Zhongjin Yang, Department of Anesthesiology, SUNY Address: Upstate Medical University, Syracuse, New York, 13210, Tel :315- 464-4890; Fax :315- 464-9930; Email : yangz@upstate.edu
\end{abstract}

\begin{abstract}
An 82-year-old female with past medical history significant for hypertrophic obstructive cardiomyopathy, atrial fibrillation and lymphoma underwent right hip arthroplasty. Aggressive fluid resuscitation prior to and during surgery was administered. At the completion of surgery, patient developed hypotension despite continuous fluid resuscitation and vasopressors infusion. The patient was found to have distended abdomen and emergent exploratory laparotomy was performed. Significantly elevated abdominal pressure $(50 \mathrm{mmHg})$ and large amount ascites $(1000 \mathrm{ml})$ were revealed. Patient's hemodynamics was immediately improved after the abdomen was opened. A diagnosis of acute abdomen compartment syndrome was made. Possible contributory factors in this patient were discussed.
\end{abstract}

\section{Introduction}

Total hip arthroplasty (THA) is one of the most successful orthopedic procedures performed today. It is estimated that over 1 million THA procedures are performed worldwide annually with high satisfaction rate ${ }^{1}$. Common early complications of this procedure include intraoperative fracture, dislocation, nerve injury, and deep vein thrombosis and pulmonary embolism. Introabdominal hypertension (IAH) and abdominal compartment syndrome (ACS) are caused by elevated intra-abdominal pressure (IAP). ACS has detrimental effects on all organ systems and is almost uniformly fatal if left untreated. To our knowledge, no published reports exist of development of ACS following a THA procedure. Here, we present a case of an elderly patient, who presented with a right hip fracture. The patient underwent a THA procedure and developed acute ACS immediately following the procedure.

\section{Case Description}

The patient is an 82-year-old female who presented to the hospital for persistent right hip pain after a fall. A right femur fracture was diagnosed and the patient was admitted for a THA.
Her past medical history included hypertrophic obstructive cardiomyopathy with outflow obstruction of 15 years duration, atrial fibrillation of unknown duration, lymphoma diagnosed in 2003, and osteoporosis. The patient appeared cachectic and was 4'5" tall and $42 \mathrm{~kg}$ body weight. Cardiology consultation was obtained and the patient was determined to be at moderate risk of morbidity. Aggressive fluid resuscitation was also advised by cardiologist to optimized intravascular volume in order to maintain hemodynamic stability. Intravenous access was a right chest port that was previously inserted for chemotherapy. A left radial arterial line was placed before induction. Following preoxygenation, the patient was induced with etomidate, fentanyl and rocuronium. The patient was intubated and anesthesia was maintained with sevoflourane. The patient then was placed on the "pegboard" table in the left decubitus position, supported by 3 posts. The inferior anterior post was placed at the level of the anterior superior iliac spine. The superior anterior post was placed over the chest wall immediately below the breast. The posterior post was placed in the sacral area. Care was taken to allow adequate ventilation. The patient was placed on volume controlled ventilation. The patient was hemodynamically stable and the surgery started. Throughout the procedure, the patient was resuscitated with $500 \mathrm{ml}$ of albumin, $3500 \mathrm{ml}$ of normal saline and 3 units of packed red blood cells for an estimated blood loss of about 1300-1500 ml. The urine output was $375 \mathrm{ml}$. The procedure itself lasted approximately 1 and a half hours. Her blood pressure and heart rate remained near her baseline level, her $\mathrm{SpO}_{2}$ was near $100 \%$ with a $\mathrm{FiO}_{2}$ of 0.6 . Towards the end of the procedure, the patient's systolic blood pressure was noticed to progressively decrease to the $70 \mathrm{~s} \mathrm{mmHg}$. In response, intermittent bolus of phenylephrine was administered (a total of 1600 micrograms) with minimal effect. Cardiology consultation was immediately obtained and vasopressin and norepinephrine drips were started. The surgery was soon finished and the patient was transferred to the post-anesthesia care unit remained intubated. Despite maximum doses of vasopressin and 
norepinephrine, the patient remained hypotensive. Her peak airway pressure was noticed to be significantly increased (from $30 \mathrm{cmH}_{2} \mathrm{O}$ to greater than $40 \mathrm{cmH}_{2} \mathrm{O}$ ). Arterial blood gas revealed severe acidosis and a total of $200 \mathrm{mEq}$ of sodium bicarbonate were administered. A transesophageal echocardiograph revealed under filled left ventricle with normal contraction. She was given additional 5L of crystalloids and 2 units of packed red blood cells. Despite the interventions, her mean arterial pressure continued to be in the low $60 \mathrm{~s} \mathrm{mmHg}$. The decision was made to place a left internal jugular cordis under ultrasound guidance. Soon after the cordis placed, the patient was noted to have facial congestion. It was thought to be due to vascular obstruction caused by the combination of the right chest port and the recent placed cordis. The cordis was immediately removed, and a decision was made to place a femoral central venous catheter. Upon exposing the abdomen and the groin area, significant abdominal distension was noticed. At this time, she developed bradycardia, with heart rate ranging from 30 to $60 \mathrm{bpm}$. An urgent general surgical consultation was obtained. Intraabdominal hemorrhage was suspected and the patient was brought back to the operating room and exploratory laparoscopy was emergently performed. Her peak airway pressures were as high as $42 \mathrm{mmHg}$. Upon the insertion of the Veress needle, her abdominal pressures measured between 30 to $50 \mathrm{mmHg}$. When a trocar was inserted, there was expulsion of small intestine and an enormous amount of yellow clear ascites. No bleeding was noted and the small and large intestines appeared to be normal. Immediately upon aspirating a significant amount of the ascetic fluid, which was approximately $1000 \mathrm{ml}$, her hemodynamics improved dramatically. Vasopressin and norepinephrine drips were stopped within minutes. Her systolic blood pressure remained close to $120 \mathrm{mmHg}$ and her peak airway pressures decreased to $30 \mathrm{mmHg}$. A total of 1100 $\mathrm{mL}$ crystalloids was given during this procedure. A diagnosis of abdominal hypertension and ACS was made. The patient was extubated next day uneventfully.

\section{Discussion}

Our patient developed significant high intraabdominal pressure (30 to $50 \mathrm{mmHg}$ ) with compromised cardiovascular function during THA. Diagnosis of IAH and ACS is appropriate. Compartment syndrome occurs when the pressure within a closed anatomic space (a compartment) becomes so elevated that organs perfusion is compromised and organs damage develops. Clinical manifestations and diagnosis criteria have been well documented by HYPERLINK "http://www.wsacs.org/" World Society of Abdominal Compartment Syndrome (WSACS) [2]. Significantly increased IAP and the development of ACS if left undetected and untreated, multisystem organ failure and patient death may be unavoidable. Risk Factors associated with IAH and ACS have also been summarized as below [3].

1. Risk Factors for IAH/ACS

- Diminished abdominal wall compliance

- Acute respiratory failure, especially with elevated intrathoracic pressure

- Abdominal surgery with primary fascia or tight closure

- Major trauma/burns

- Prone position, head of bed $>30$ degrees

- High body mass index (BMI), central obesity

- Increased intro-luminal pressure

- Gastroparesis

- Ileus

- Colonic pseudo-obstruction

2. Increased abdominal contents

3. Hemoperitoneum/ pneumoperitoneoum

4. Ascites/liver dysfunction

5. Capillary leak/fluid resuscitation

- Acidosis $(\mathrm{pH}<7.2)$

- Hypotension

- Hypothermia (core temperature $<33^{\circ} \mathrm{C}$ )

- Polytransfuion (> 10 units of blood /24 hours)

- Coagulopathy (Platelets $<55.000 \mathrm{~mm}^{3}$ or PT $>15$ seconds or PTT $>2$ times normal or INR $>1.5$

- Massive fluid resuscitation (5L/24 hours)

- Pancreatitis

- Oliguria

- Sepsis

- Major trauma/Burns

- Damage control Laparotomy

A normal IAP is $<5$ to $7 \mathrm{mmHg}$ (with the upper limit of 12 $\mathrm{mmHg}$ ) according to the consensus definition of the WSACS in healthy individuals. In contrast, a constant IAP greater than $12 \mathrm{mmHg}$ defines IAH. IAH may be conveniently divided into 4 grades that are further subdivided according to the rapidity of onset. The grades range from grade $1(12-15 \mathrm{~mm} \mathrm{Hg})$ to grade II (16-20 $\mathrm{mm} \mathrm{Hg}$ ) to grade III (20-24 $\mathrm{mm} \mathrm{Hg}$ ) to grade IV ( $>25$ $\mathrm{mm} \mathrm{Hg}$ ); the onset times range from chronic to acute to subacute to hyperacute [4]. Our patient developed hyperacute and grade IV ACS. Exact etiology caused IAH and the development of ACS in our patient is unclear, multiple factors may be responsible. One possibility is that the position may cause diminished abdominal wall compliance. The patient was placed decubitus position on the pegboard operating table stabilized by three posts. Considered cachectic status and short statue of our patient, it is possible that one of the posts pressed on the lower chest wall such that it might compress the inferior vena cava. This would lead to a decreased venous return, as evidenced by 
bedside Echocardiogram showing under filled left ventricle with normal contraction. Diminished pre-load reduced cardiac output and resulted in sustained hypotension. Compressed inferior vena cava also caused intrahepatic pressure to rise. Increased intrahepatic pressure would cause extravasation of fluid into the abdominal cavity, causing ascites, which would cause increased abdominal pressure, worsening inferior vena cava obstruction, led to the lower limbs edema. Aggressive fluid resuscitation may be another contributory factor. The reason for aggressive fluid resuscitation prior to and during the procedure was based on the patient's medical conditions. She was cachectic with hypertrophic obstructive cardiomyopathy with outflow obstruction. Adequate hydration prior to and during the surgery would be important to maintain an adequate cardiac output. Throughout the procedure, the patient received

total $500 \mathrm{ml}$ of albumin, $3500 \mathrm{ml}$ of normal saline and 3 units of packed red blood cells. Considered this patient's body weight (42 kg), fluid resuscitation seemed too aggressive. High-volume fluid resuscitation ( $>3500 \mathrm{ml} / 24 \mathrm{~h}$ ) is known a risk factor for increased IAP [3]. In our patient, due to the obstruction of inferior venous cava, resuscitated fluid would stay and cumulated in the lower body parts. This would cause abdominal organs congestion and the fluid may leak out as acute ascites which further increased IAP. Increased IAP caused poor abdominal organs perfusion, which leads to metabolic acidosis as seen in this patient. Acidosis increased capillary permeability and caused more fluid leak out. This vicious cycle eventually caused organs damage and resulted to the development of ACS. ACS follows a destructive pathway similar to the limb compartment syndrome with a high mortality rate if left untreated. Our patient was brought back to OR and her condition was rapidly improved immediately after decompression, suggesting that early reorganization of IAH is essential to avoid the development of ACS. To our knowledge, there has not been a case of ACS reported after a total hip arthoplasty. Our case illustrates that rare complications can occur in cases that involve common and relatively successful procedures. As Cheatham pointed out that all clinicians should be aware of the risk factors that predict development of IAH/ACS, the appropriate measurement of IAP, and the current resuscitation options for managing these highly morbid syndromes [3]. This case suggests that ACS may be considered if a sudden cardiovascular collapse associated with distended abdomen.

\section{References}

1. Del Buono Angelo, Denaro, V., Maffulli,N.(2012). Genetic susceptibility to aseptic loosening following total hip arthroplasty: a systematic review. British Medical Bulletin, 101, 39-55.

2. http://www.IntraAbdominalHypertension.org

3. Cheatham, M. L. (2009). Nonoperative management of intraabdominal hypertension and abdominal compartment syndrome. World Journal of Surgery, 33, 1116-22.

4. Maerz, L., Kaplan, L.J. (2008). Abdominal compartment syndrome. Critical Care Medicine, 36 [Suppl], S212-S215. 